CARDIOVASCULAR MEDICINE

\title{
Effects of ventricular rate and regularity on the velocity and magnitude of left atrial appendage flow in atrial fibrillation
}

\author{
O A Obel, L Luddington, N Maarouf, K Aytemir, C Ekwall, M Malik, A J Camm
}

Heart 2005;91:764-768. doi: 10.1136/hrt.2003.030940

See end of article for authors' affiliations

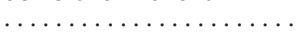

Correspondence to: Dr Owen A Obel,

Department of Cardiology $111 \mathrm{~A}$, Dallas VA Healthcenter, 4500 South

Lancaster Road, Dallas, TX

75216, USA; owen.obel@ utsouthwestern.edu

Accepted 7 July 2004

\begin{abstract}
Objective: To prospectively determine whether ventricular rate and regularity are significant determinants of the velocity and magnitude of left atrial appendage (LAA) flow.

Design and patients: 12 patients with atrial fibrillation (AF), high degree atrioventricular block, and indwelling permanent pacemakers were studied.

Setting: Cardiology department of a tertiary referral centre.

Interventions: Pacing was triggered by an external programmable transcutaneous device. Patients were paced at 60,120 , and 150 beats/min in both regular and irregular rhythm. LAA flow velocity and magnitude were assessed with transoesophageal Doppler echocardiography.

Main outcome measures: Peak and mean LAA inflow and outflow velocity, and time-velocity interval (TVI) of LAA flow.

Results: Increasing ventricular rate was associated with significantly lower peak inflow ( $p<0.01$ ), peak outflow ( $p<0.05)$, mean inflow $(p<0.01)$, and mean outflow $(p<0.05)$ velocities and with a lower TVI of LAA filling and emptying velocities $(p<0.01)$. This effect was noted at rates of 60 beats $/ \mathrm{min}$ compared with both 120 and 150 beats $/ \mathrm{min}$. At a pacing rate of 120 beats $/ \mathrm{min}$ there was a significantly higher total TVI when pacing at a regular than at an irregular rhythm $(40.16(14.6) \mathrm{cm} v 30.74(10.9) \mathrm{cm}$, $\mathrm{p}<0.05)$.

Conclusions: In this study, LAA filling velocities in patients in AF were significantly influenced by paced ventricular rate and to a much lesser extent ventricular rhythm. These results suggest that rapid ventricular rates may predispose to stasis in the LAA in AF.
\end{abstract}

A trial fibrillation $(\mathrm{AF})$ is associated with an increased risk of systemic embolism and stroke, even in the absence of valvar heart disease. ${ }^{1}$ The majority of these events result from thrombus formation within the left atrium, in particular the left atrial appendage (LAA). ${ }^{2}$ Echocardiographic risk factors for LAA thrombus and systemic embolism include decreased LAA flow velocity and the observation of spontaneous echo contrast (SEC) within the left atrium and LAA. ${ }^{34}$

LAA flow has been shown to be related to left ventricular (LV) filling and loading, ${ }^{5}$ which in turn are related to ventricular rate and regularity of rhythm. ${ }^{67}$ Furthermore, the LAA lies within the confines of the pericardium close to the LV free wall. The purpose of this study was to prospectively determine, in a controlled manner, whether ventricular rate and regularity influence the velocity and magnitude of LAA flow.

\section{PATIENTS AND METHODS \\ Patients}

The study group comprised 12 patients aged 43-74 years with high degree atrioventricular (AV) block who were in AF. Five patients had undergone AV nodal ablation, most recently 16 months before the study. All patients had an indwelling permanent pacemaker with the facility to be programmed to triggered (VVT) mode. Full ethical approval was obtained for the study and all patients gave informed written consent. Potential study participants were identified by reviewing records from our pacemaker follow up clinic and records of AV nodal ablation procedures. Patients with permanent pacemakers implanted for high degree AV block who had a history of AF were identified. Patients were excluded if they had significant mitral valve disease; if they were not able to safely tolerate brief periods of rapid ventricular pacing; or if they were unable to undergo transoesophageal echocardiography because of failure to tolerate a semi-recumbent left lateral position or because of oesophageal disease. Patients with paroxysmal AF were included only if they were in AF at the time of the study.

Patients fasted for at least four hours before the study and were monitored during the study protocol by three precordial unipolar surface ECG leads, manual sphygmomanometric blood pressure monitoring updated each two minutes, and continuous pulse oximetry. After peripheral venous access was secured, patients were studied in the semi-recumbent, supine position.

\section{Pacing}

All patients were in a paced ventricular rhythm for the entire study. To examine the effects of ventricular rate and rhythm on parameters of LAA flow, the indwelling pacemaker was temporarily reprogrammed to unipolar VVT (ventricular triggered) mode at a base rate of 40 beats/min. Rate and rhythm were modified with an electrically isolated programmable device delivering $5 \mathrm{~V}$ monophasic transcutaneous signals through two surface skin electrodes. With the indwelling pacemaker programmed to VVT mode, pacing responds to sensed events, thus allowing the external device to control the ventricular rate and rhythm reliably. The system was tested in the run-up to the study and was found to consistently trigger the indwelling pacemaker. By this method, ventricular rate and rhythm could be rapidly and precisely adjusted. The transcutaneous signals did not directly activate skeletal or cardiac muscle and no patient

Abbreviations: $A F$, atrial fibrillation; $A V$, atrioventricular; $L A A$, left atrial appendage; LV, left ventricular; SEC, spontaneous echo contrast; TVI, time-velocity interval 


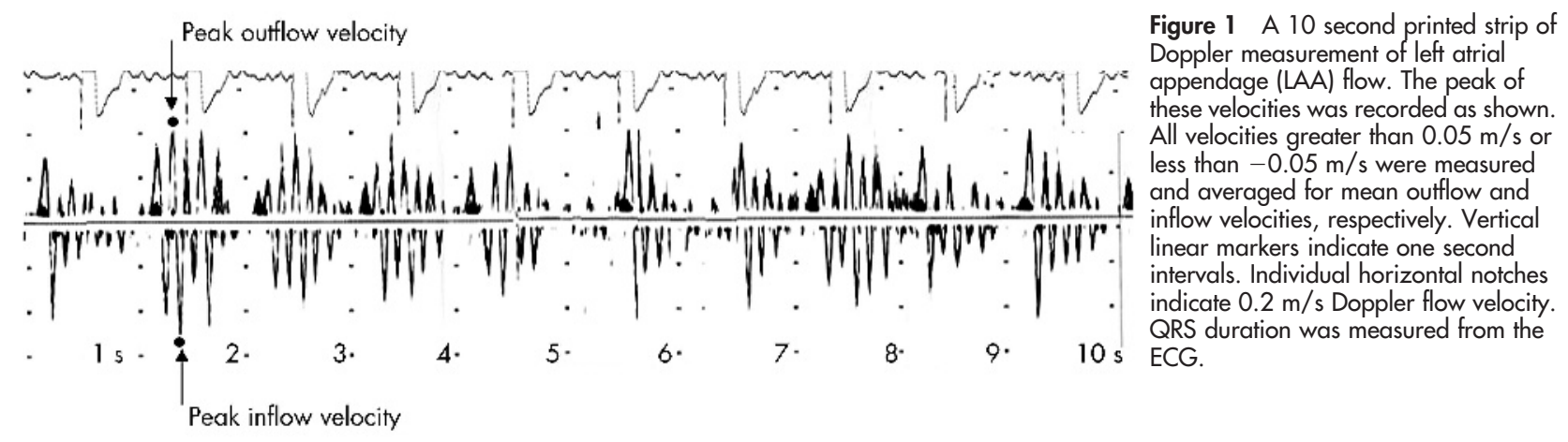

reported more than a mild tingling sensation in response to the transcutaneous signals.

The device was programmed to deliver regular sequences and irregular sequences simulating AF. The character of the irregular sequence-that is, the proportions between consecutive intervals - was derived directly from a rhythm strip of a patient in AF at a rate of about 60 beats/min. Each patient was paced for three minutes in each sequence. Thus, the pacing sequences were as follows: pattern 1, 60 beats/min in regular rhythm; pattern 2, 60 beats/min in irregular rhythm; pattern 3, 120 beats/min in regular rhythm; and pattern 4,120 beats/min in irregular rhythm. In addition, eight patients were paced at 150 beats/min (pattern 5). In four patients (two with dilated cardiomyopathy, one with ischaemic heart disease, and one with lone AF) we decided not to pace at 150 beats/min, since we felt this to be clinically undesirable. Pacing at 150 beats/min was performed only in regular rhythm.

\section{Echocardiography}

Intravenous sedation with 2-5 mg midazolam was administered to all but one patient and local anaesthesia with $1 \%$ lidocaine spray was applied to the pharynx. An omniplane $5 \mathrm{MHz}$ transoesophageal probe (Hewlett Packard Ltd) was passed into the oesophagus and the probe was flexed. Two dimensional views were used to exclude left atrial and appendageal thrombus and significant mitral regurgitation. The LAA and its orifice were viewed in the basal short axis plane and the Doppler sample volume was positioned at the orifice. Once stable pulsed Doppler recordings of LAA inflow and outflow were made, the pacing protocol was commenced.

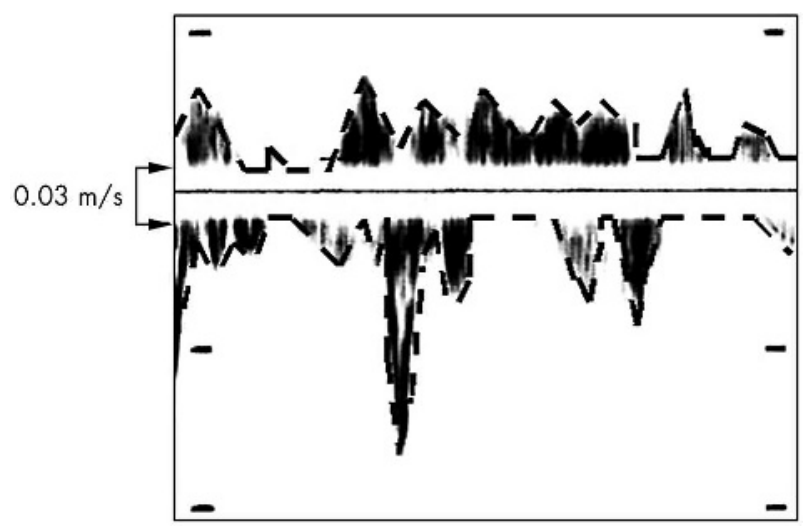

Figure 2 Time-velocity interval (TVI) was measured by tracing a digital pen over the Doppler signal printout for the 10 second period. The filtered portion above and below the true zero line $10.03 \mathrm{~m} / \mathrm{s}$ on either side) was not included in the digital tracing, which was passed over a straight ruler at that point.
After 150 seconds in each pacing sequence, 10 seconds of pulsed Doppler recordings of LAA flow were stored on videotape. Following this, a 10 second printout of Doppler flow was made for the measurement of LAA flow velocities and time-velocity interval (TVI) of LAA flow.

From each printout, three measurements were made: firstly, the tallest and deepest signal were recorded as peak velocities of LAA outflow and inflow, respectively; secondly, all signals during the 10 second period that were greater than $0.05 \mathrm{~m} / \mathrm{s}$ (outflow) or less than $-0.05 \mathrm{~m} / \mathrm{s}$ (inflow) were recorded and averaged to give mean velocities of LAA outflow and inflow, respectively (fig 1); and lastly, TVI was measured by tracing the area under the curve for the 10 second period with a digital pen (fig 2). The values for TVI of LAA outflow and inflow were added together to obtain total TVI. This measurement provides an estimate of the magnitude of LAA flow, assuming a constant diameter of the LAA orifice.

The presence or absence of SEC was noted during each pacing protocol. QRS duration was measured manually from the ECG printed at the time of pulsed Doppler recording of LAA flow (fig 1). After transoesophageal echocardiography, a transthoracic echocardiogram was recorded with the patient in the left lateral position, paced at 60 beats/min in a regular rhythm. Ejection fraction was calculated from $M$ mode measurements of ventricular diameters at end systole and end diastole acquired in the parasternal long axis view.

\section{Analysis of results}

Repeated measures analysis of variance was used to compare continuous dependent variables according to either the pacing rate used or the use of irregular pacing cadence. Pacing patterns were compared by analysis of variance with Bonferroni correction. Differences between patterns are presented as mean (SD). An adjusted $\mathrm{p}<0.05$ was considered significant.

\section{RESULTS}

Twelve patients with a mean age of 61. 6 years and a mean AF duration of 6.8 years were studied. Seven patients had lone AF (table 1). Mean ejection fraction was $46.2 \%$ (range $22-60 \%$ ) and one hypertensive patient had mild LV hypertrophy. The study group was therefore younger than the general AF population, with a preponderance of patients with lone $\mathrm{AF}$ - that is, occurring in the absence of hypertension or structural heart disease.

Ventricular rate had a significant effect on peak inflow $(p<0.01)$ and outflow $(p<0.05)$ and on mean inflow $(\mathrm{p}<0.01)$ and outflow $(\mathrm{p}<0.05)$ velocities (table 2$)$. Similarly, ventricular rate had a significant effect on inflow, outflow, and total TVI $(\mathrm{p}<0.01)$ (fig 3 ). This was only noted when pacing at 60 beats/min was compared with pacing at 120 and 150 beats/min (figs 4 and 5). 
Table 1 Characteristics of the study patients

\begin{tabular}{|c|c|c|c|c|c|c|c|c|}
\hline Patient & Age (years) & Sex & $\mathbf{A F}$ & $\begin{array}{l}\text { AF duration } \\
\text { (years)* }\end{array}$ & $\begin{array}{l}\text { Underlying cardiac } \\
\text { condition }\end{array}$ & Pacemaker mode & $\begin{array}{l}\text { PPM implanted } \\
\text { (years) }\end{array}$ & $\begin{array}{l}\text { Baseline QRS duration } \\
\text { (ms) }\end{array}$ \\
\hline 1 & 54 & $M$ & Permanent & 5.5 & DCM & VVI & 3 & 190 \\
\hline 2 & 68 & $M$ & Permanent & 12 & DCM & $\mathrm{VI}$ & 10 & 160 \\
\hline 3 & 58 & $M$ & Permanent & 3 & Lone & VI & 1 & 280 \\
\hline 4 & 64 & $M$ & Paroxysmal & 4 & Lone & DDD & 1.5 & 280 \\
\hline 5 & 74 & $\mathrm{~F}$ & Permanent & 7.5 & IHD & VVI & 2 & 150 \\
\hline 6 & 59 & $\mathrm{~F}$ & Permanent & 4.5 & Lone & $\mathrm{VI}$ & 2.5 & 170 \\
\hline 7 & 45 & $M$ & Paroxysmal & 3 & Lone & DDD & 1 & 170 \\
\hline 8 & 62 & $M$ & Permanent & 6.5 & Hypertension & $\mathrm{VI}$ & 3 & 162 \\
\hline 9 & 51 & $\mathrm{~F}$ & Permanent & 4 & Lone & $\mathrm{VI}$ & 2 & 160 \\
\hline 10 & 65 & $M$ & Permanent & 5.5 & Lone & $\mathrm{VI}$ & 1.5 & 170 \\
\hline 11 & 71 & $M$ & Permanent & 18 & Hypertension & VI & 2 & 200 \\
\hline 12 & 68 & $\mathrm{~F}$ & Permanent & 9 & Lone & $\mathrm{VI}$ & 3.5 & 160 \\
\hline
\end{tabular}

*Six month approximation.

$A F$, atrial fibrillation; $D C M$, dilated cardiomyopathy; $F$, female; IHD, ischaemic heart disease; lone $A F$, atrial fibrillation in the absence of hypertension or structural heart disease; $M$, male; PPM, permanent pacemaker.

Despite a trend towards lower mean inflow velocities with irregular pacing $(p=0.08)$, regularity of rhythm did not produce a significant effect on LAA flow velocities or almost all measurements of TVI. At a rate of 120 beats/min there was a significantly higher total TVI when pacing in a regular than with an irregular rhythm $(p<0.05)$. SEC within the left atrium and LAA was noted in four of the patients at baseline. In two other patients, SEC was noted during ventricular pacing at 150 beats/min.

Exclusive right ventricular pacing at 60 beats/min resulted in a prolonged baseline QRS duration in all patients (187 (45) ms). Neither ventricular rate nor rhythm had a significant effect on QRS duration, although there was some variability in QRS duration when pacing was irregular.

\section{DISCUSSION}

The main findings of the present study are that LAA flow during AF has a significantly higher velocity and a greater magnitude at slower than at faster ventricular rates. The effect was significant when 60 beats/min was compared with 120 and 150 beats/min but not when 120 beats/min was compared with 150 beats/min. Irregular ventricular rhythm had much less of an effect than did ventricular rate on standard LAA flow indices.

Results of two previous studies have suggested that ventricular rate during AF may be an important determinant of LAA flow. Akosah and colleagues ${ }^{8}$ studied 26 patients with $\mathrm{AF}$ before and after cardioversion and found that LAA ejection fraction was inversely related to ventricular rate in AF but not in sinus rhythm. Furthermore, they noted that the occurrence of SEC or atrial thrombus was related to higher ventricular rates. Noda and colleagues ${ }^{9}$ studied 21 patients with non-valvar AF and analysed LAA flow in relation to heart rate by averaging 3-5 consecutive beats consisting of short RR intervals and 10 beats of long RR intervals. Average peak velocity of LAA flow was higher during longer RR intervals and during LV diastole than during LV systole. LAA flow has not been prospectively studied in relation to the controlled and sustained manipulation of ventricular rate and rhythm.

Stroke in patients with AF arises largely as a result of thromboembolism from the LAA. There is strong evidence that stasis of LAA flow is a major risk factor for the occurrence of LAA thrombus. ${ }^{2-4}$ Ventricular function is known to be a major determinant of both LAA flow and thromboembolic risk. ${ }^{10-13}$ As heart rate increases, diastolic filling time proportionately decreases to a far greater degree than ventricular systole and is very short when heart rate is rapid. ${ }^{14}{ }^{15}$ The relation of LAA and LV function has been studied in most detail in patients who were in sinus rhythm. ${ }^{5}$ It was shown that LV diastole is closely linked to the early portion of LAA emptying and, when shortened, may have an impact on LAA flow. Patients with AF characteristically lack a late diastolic ventricular filling $(\mathrm{A})$ wave since it is dependant on atrial systole ${ }^{16}$ and therefore extrinsic influences may play an even larger part in LAA function. The effects of LV diastolic filling on LAA flow may assume increased

Table 2 Effect of ventricular rate and regularity on left atrial appendage flow parameters

\begin{tabular}{|c|c|c|c|c|c|}
\hline \multirow[b]{2}{*}{ Parameter } & \multicolumn{5}{|l|}{ Pacing pattern } \\
\hline & $1(n=12)$ & $2(n=12)$ & $3(n=12)$ & $4(n=12)$ & $5(n=8)$ \\
\hline Peak inflow $(\mathrm{m} / \mathrm{s})$ & $0.48(0.2)^{*}$ & $0.45(0.22)^{*}$ & $0.4(0.21)$ & $0.36(0.18)$ & $0.3(0.13)$ \\
\hline Peak outflow $(\mathrm{m} / \mathrm{s})$ & $0.47(0.19)^{* *}$ & $0.5(0.2)$ & $0.37(0.19)$ & $0.4(0.17)$ & $0.37(0.24)$ \\
\hline Mean inflow $(\mathrm{m} / \mathrm{s})$ & $0.36(0.13)^{*}$ & $0.32(0.17)^{*}$ & $0.23(0.11)$ & $0.21(0.08)$ & $0.19(0.08)$ \\
\hline Mean oufflow $(\mathrm{m} / \mathrm{s})$ & $0.33(0.13)^{\star *}$ & $0.32(0.17)^{*}$ & $0.23(0.12)$ & 0.21 (0.09) & $0.21(0.1)$ \\
\hline TVI inflow $(\mathrm{cm})$ & $30.76(14.2)^{*}$ & $28.9(13.1)^{*}$ & $22.05(8.65)$ & $18.9(8.15)$ & $16.3(8)$ \\
\hline TVI outflow $(\mathrm{cm})$ & $31.88(13.2)^{*}$ & $25.01(10.3)^{*}$ & $18.13(7.6)$ & $17.21(6.6)$ & $14.74(8.8)$ \\
\hline TVI total flow $(\mathrm{cm})$ & $62.39(23.5)^{*}$ & $53.93(20.7)^{*}$ & $40.16(14.6) t$ & 30.74 (10.9) & $32.16(14.1)$ \\
\hline QRS duration (ms) & $187(45)$ & $187(45)$ & $177(21)$ & $177(21)$ & $170(25)$ \\
\hline Ejection fraction (\%) & $46.2(11.4)$ & NA & NA & NA & NA \\
\hline LVIDs (mm) & $40(9.7)$ & NA & NA & NA & NA \\
\hline LVIDd (mm) & $53(7.1)$ & NA & NA & NA & NA \\
\hline \multicolumn{6}{|c|}{$\begin{array}{l}\text { Data are mean (SD). } \\
\text { * } \mathrm{p}<0.01 \text { versus patterns } 3,4 \text {, and } 5 ;{ }^{* *} \mathrm{p}<0.05 \text { versus patterns } 3,4 \text {, and } 5 ; \mathrm{tp}<0.05 \text { versus pattern } 4 . \\
\text { Pacing pattern 1: } 60 \text { beats/min, regular rhythm; pattern } 2: 60 \text { beats/min, irregular rhythm; pattern } 3: 120 \text { beats/ } \\
\text { min, regular rhythm; pattern } 4: 120 \text { beats/min, irregular rhythm; pattern } 5: 150 \text { beats/min, regular rhythm. } \\
\text { LVIDs, left ventricular internal diameter in systole; LVIDd, left ventricular internal diameter in diastole; NA, not } \\
\text { applicable; TVI, time-velocity interval. }\end{array}$} \\
\hline
\end{tabular}




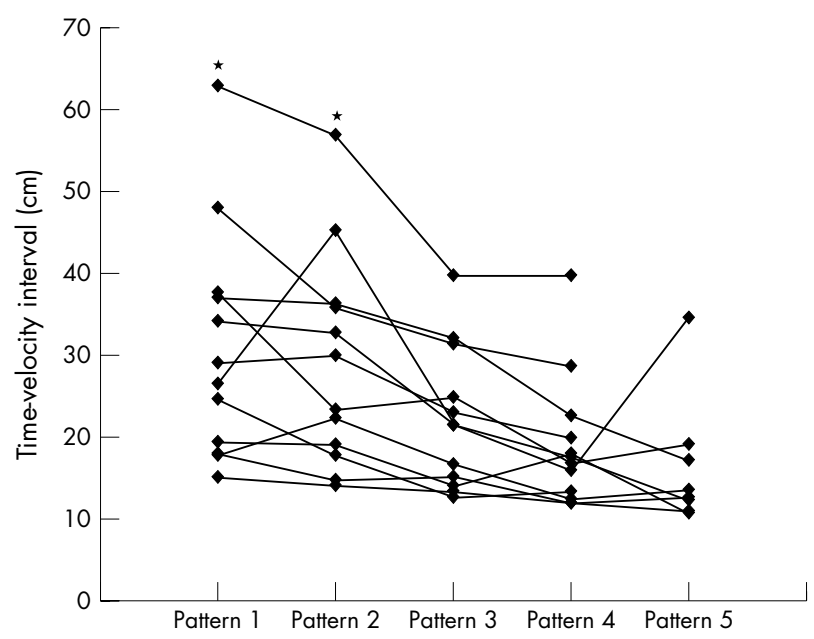

Figure 3 TVI plotted according to pacing pattern. Patients under pacing patterns 1 ( 60 beats/min, regular rhythm) and 2 (60 beats/min, irregular rhythm) had significantly higher peak inflow velocities than under patterns 3 (120 beats/min, regular rhythm), 4 (120 beats/min, irregular rhythm), and 5 (150 beats $/ \mathrm{min}$, regular rhythm). ${ }^{*} \mathrm{p}<0.01$.

importance when AF is longstanding and intrinsic appendageal contraction is impaired. ${ }^{17}$ The inverse relation between paced ventricular rate and LAA flow was significant in comparisons of 60 versus 120 beats/min but not in comparisons of 120 versus 150 beats/min. This suggests that a lower limit of diastolic filling time is reached at a heart rate of 120 beats/min, below which there is no effect on LAA flow. In addition to the intracavitary effects of LV function on LAA flow, the inferomedial portion of the LAA may be externally compressed during ventricular diastole between the LV free wall and the pericardium.

The irregular ventricular rhythm that accompanies AF is associated with a decrease in cardiac output and a subsequent increase in atrial pressure. ${ }^{67}$ This reduction in ventricular function associated with an irregular ventricular rhythm may affect LAA flow. The reasons for the comparative lack of an effect of an irregular ventricular rhythm on LAA flow in this study may relate to longer RR intervals compensating for the effects of the shorter intervals on ventricular diastole.

\section{Clinical implications}

Optimisation of ventricular rate control may be a means of reducing thromboembolic risk in patients with persistent AF.
As a result of recent randomised controlled studies of patients with persistent AF, physicians may be increasingly more likely to choose rate control as a strategy in such instances. ${ }^{18} 19$ This can be achieved with medication or with AV node ablation and permanent ventricular pacing. ${ }^{20}$

The effects of paced ventricular rate and regularity on LAA function were generally more pronounced on TVI of LAA filling velocities than on peak and mean velocities. TVI has not been previously used in the estimation of LAA flow and the results of this study indicate that it may be an additional method of quantifying thromboembolic risk.

\section{Study limitations}

The small number of patients in the study tends to limit the interpretation of the results and make subgroup analysis difficult. However, the power of the study is increased by the fact that patients acted as their own controls. By necessity, the study was conducted with patients placed in the semirecumbent position. Whether similar effects would be observed in ambulatory patients is unknown. The study examined the short term effects of ventricular pacing on LAA flow. It is not certain that similar effects would be present in the long term. Baseline measurements of LAA flow suggested that this group of patients was not within a high risk for thromboembolism by this criterion, since velocities of $<0.25 \mathrm{~m} / \mathrm{s}$ are generally thought to identify high risk patients. It is not known whether LV rate would affect LAA flow in patients with lower baseline velocities.

All patients in the study were right ventricular paced, which causes dyssynchronous ventricular function manifested by a broad QRS complex. This may have a significant effect on LV filling, particularly at higher rates. The width of the QRS did not change significantly at faster pacing rates, thereby standardising this effect. Although QRS duration was measured in a single lead only, this was constant for each patient. It is not known whether patients who have synchronous ventricular activation (either at baseline or as a result of biventricular pacing) exhibit similar effects of ventricular rate on LAA flow. The effects of LV function on LAA flow are mediated through the effect of cardiac output on atrial pressure, neither of which could be measured during this study. The diameter of the LAA orifice was not measured during different pacing protocols; therefore, TVI is not a precise representation of the magnitude of LAA flow.

\section{Conclusion}

This study shows that ventricular pacing rate is an important determinant of the velocity and magnitude of LAA flow in patients with AF. The results of this study suggest that, in

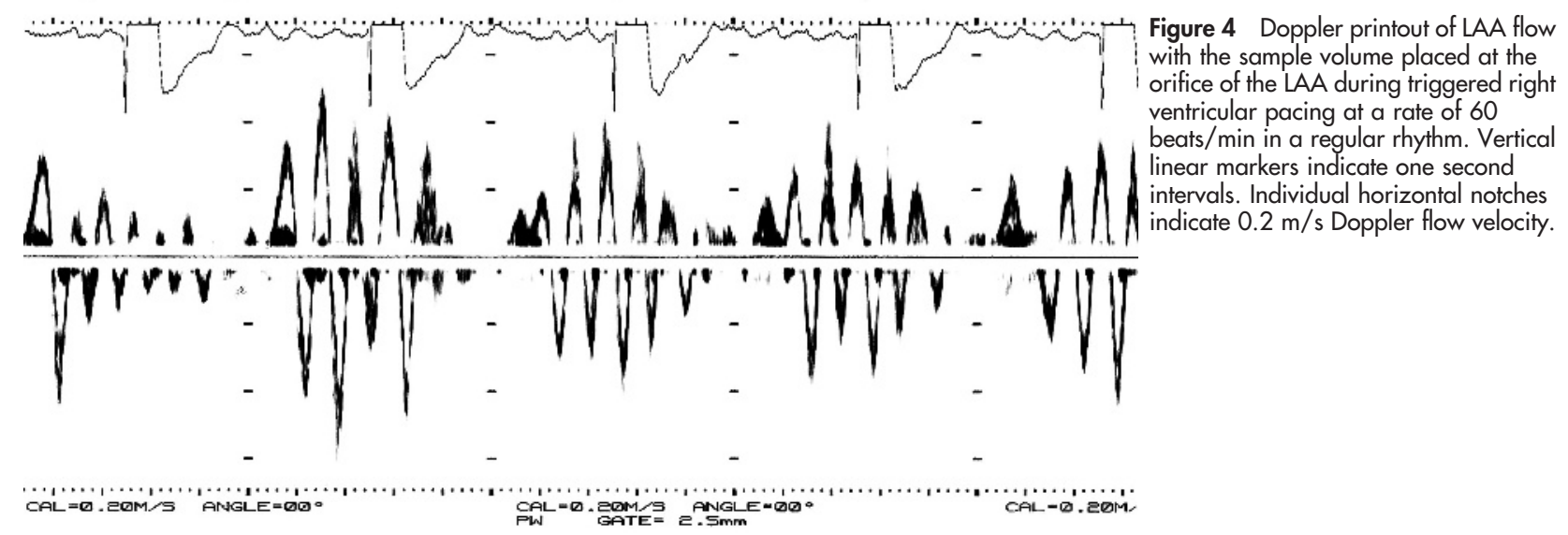




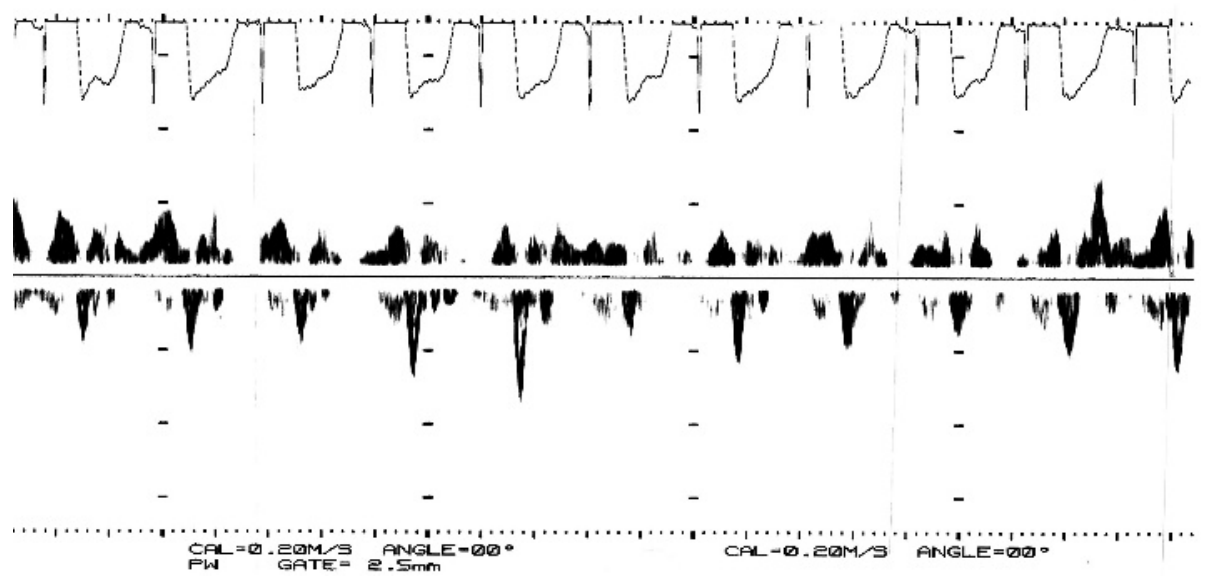

Figure 5 Doppler printout of LAA flow at a rate of 150 beats $/ \mathrm{min}$ in a regular rhythm. Note the reduced peak and overall velocity of LAA inflow and outflow when the right ventricle is paced at this faster rate. Vertical linear markers indicate one second intervals. Individual horizontal notches indicate $0.2 \mathrm{~m} / \mathrm{s}$ Doppler flow velocity

addition to relief of symptoms and prevention of tachycardia related cardiomyopathy, improved ventricular rate control may be a means of reducing thromboembolic risk in AF. These effects were significant when a ventricular rate of 60 beats/min was compared with 120 beats/min. Regularity of ventricular rhythm affected measurements of LAA flow to a much lesser degree.

\section{Authors' affiliations}

O A Obel, L Luddington, N Maarouf, K Aytemir, C Ekwall, M Malik, A J Camm, Department of Cardiological Sciences, St Georges Hospital Medical School, London, UK

\section{REFERENCES}

1 Wolf PA, Abbott RD, Kannel WB. Atrial fibrillation as an independent risk factor for stroke: the Framingham study. Stroke 1991;22:983-8.

2 Manning WJ, Silverman DI, Keighley CS, et al. Transesophageal echocardiographically facilitated early cardioversion from atrial fibrillation using short-term anticoagulation: final results of a prospective 4.5-year study. J Am Coll Cardiol 1995;25:1354-61.

3 Fatkin D, Kelly RP, Feneley MP. Relations between left atrial appendage blood flow velocity, spontaneous echocardiographic contrast and thromboembolic risk in vivo. J Am Coll Cardiol 1994;23:961-9.

4 Zabalgoitia M, Halperin JL, Pearce LA, et al. Transesophageal echocardiographic correlates of clinical risk of thromboembolism in nonvalvular atrial fibrillation. SPAF III Investigators. J Am Coll Cardiol 1998;31:1622-6.

5 Kortz RA, Delemarre BJ, van Dantzig JM, et al. Left atrial appendage blood flow determined by transesophageal echocardiography in healthy subjects. Am J Cardiol 1993;71:976-81.

6 Naito M, David D, Michelson EL, et al. The hemodynamic consequences of cardiac arrhythmias: evaluation of the relative roles of abnormal atrioventricular sequencing, irregularity of ventricular rhythm and atrial fibrillation in a canine model. Am Heart J 1983;106:284-91.
7 Daoud EG, Weiss R, Bahu $M$, et al. Effect of an irregular ventricular rhythm on cardiac output. Am J Cardiol 1996:78:1433-6.

8 Akosah KO, Funai JT, Porter TR, et al. Left atrial appendage contractile function in atrial fibrillation: influence of heart rate and cardioversion to sinus rhythm. Chest 1995;107:690-6.

9 Noda T, Arakawa M, Miwa H, et al. Effects of heart rate on flow velocity of the left atrial appendage in patients with nonvalvular atrial fibrillation. Clin Cardiol 1996; 19:295-300.

10 Hoit BD, Shao Y, Gabel M. Influence of acutely altered loading conditions on left atrial appendage flow velocities. J Am Coll Cardiol 1994;24:1117-23.

11 Fatkin D, Feneley MP. Patterns of Doppler-measured blood flow velocity in the normal and fibrillating human left atrial appendage. Am Heart J 1996;132:995-1003.

12 Lin JM, Hsu KL, Hwang J, et al. Influence of left ventricular diastole on left atrial appendage blood flow in patients with nonrheumatic atrial fibrillation. Cardiology 1997:88:563-8.

13 SPAF Investigators. Predictors of thromboembolism in atrial fibrillation II: echocardiographic features of patients at risk. The Stroke Prevention in Atrial Fibrillation Investigators. Ann Intern Med 1992;116:6-12.

14 Cieslinski A, Hui WK, Oldershaw PJ, et al. Interaction between systolic and diastolic time intervals in atrial fibrillation. Br Heart J 1984;51:431-7.

15 Lavine SJ, Krishnaswami V, Levinson N, et al. Effect of heart rate alterations produced by atrial pacing on the pattern of diastolic filling in normal subjects. Am J Cardiol 1988;62:1098-102.

16 Pollick C, Taylor D. Assessment of left atrial appendage function by transesophageal echocardiography: implications for the development of thrombus. Circulation 1991;85:223-31.

17 Manning WJ, Silverman DI, Katz SE, et al. Impaired left atrial mechanical function after cardioversion: relation to the duration of atrial fibrillation. J Am Coll Cardiol 1994:23:1535-40.

18 Wyse DG, Waldo AL, DiMarco JP, et al. A comparison of rate control and rhythm control in patients with atrial fibrillation. N Engl J Med 2002;347:1825-33.

19 Van Gelder IC, Hagens VE, Bosker HA, et al. A comparison of rate control and rhythm control in patients with recurrent persistent atrial fibrillation. N Engl J Med 2002;347:1834-40.

20 Natale A, Zimerman L, Tomassoni G, et al. Impact on ventricular function and quality of life of transcatheter ablation of the atrioventricular junction in chronic atrial fibrillation with a normal ventricular response. Am J Cardiol 1996;78:1431-3.

\section{bmjupdates+}

bmjupdates+ is a unique and free alerting service, designed to keep you up to date with the medical literature that is truly important to your practice.

bmjupdates+ will alert you to important new research and will provide you with the best new evidence concerning important advances in health care, tailored to your medical interests and time demands.

Where does the information come from?

bmjupdates+ applies an expert critical appraisal filter to over 100 top medical journals A panel of over 2000 physicians find the few 'must read' studies for each area of clinical interest

Sign up to receive your tailored email alerts, searching access and more...

www.bmjupdates.com 